\title{
Hyperuricemia and long-term mortality in patients with acute myocardial infarction undergoing percutaneous coronary intervention
}

\author{
Wei Guo ${ }^{1,2 \#}$, Dahao Yang ${ }^{3 \#}$, Dengxuan Wu ${ }^{4 \#}$, Huixia Liu ${ }^{1}$, Shiqun Chen ${ }^{2}$, Jin Liu ${ }^{2}$, Li Lei ${ }^{2}$, Yong Liu ${ }^{2}$, \\ Lifen Rao', Li Zhang'; The RESCIND Group \\ ${ }^{1}$ Guangdong Provincial Geriatrics Institute, Guangdong Provincial People’s Hospital, Guangdong Academy of Medical Sciences, Guangzhou 510080, China; \\ ${ }^{2}$ Department of Cardiology, Guangdong provincial Key Laboratory of Coronary Heart Disease Prevention, Guangdong Cardiovascular Institute, Guangdong \\ Provincial People's Hospital, Guangdong Academy of Medical Sciences, Guangzhou 510080, China; ${ }^{3}$ Fuwai Hospital Chinese Academy of Medical Sciences, \\ Shenzhen, Shenzhen 518000, China; ${ }^{4}$ Department of Cardiology, Panzhihua Central Hospital, Panzhihua 617000, China \\ Contributions: (I) Conception and design: W Guo; (II) Administrative support: Y Liu, L Rao, L Zhang; (III) Provision of study materials or patients: \\ All authors; (IV) Collection and assembly of data: All authors; (V) Data analysis and interpretation: W Guo, Y Liu, S Chen, J Liu, L Rao, L Zhang; (VI) \\ Manuscript writing: All authors; (VII) Final approval of manuscript: All authors. \\ \#These authors contributed equally to this work. \\ Correspondence to: Yong Liu, MD, PhD. Department of Cardiology, Guangdong provincial Key Laboratory of Coronary Heart Disease Prevention, \\ Guangdong Cardiovascular Institute, Guangdong Provincial People’s Hospital, Guangdong Academy of Medical Sciences, Guangzhou 510080, \\ China. Lifen Rao, MD; Li Zhang, MD. Guangdong Provincial Geriatrics Institute, Guangdong Provincial People's Hospital, Guangdong Academy \\ of Medical Sciences, Guangzhou 510080, China. Email: liuyong2099@126.com; raodoctor@126.com; gdgdavid@163.com.
}

\begin{abstract}
Background: Although serum uric acid (UA) was regarded to be involved in cardiovascular disease, the role of serum UA (SUA) as a risk factor in acute myocardial infarction (AMI) is controversial. We investigated whether hyperuricemia was linked with long-term mortality in patients with AMI who underwent percutaneous coronary intervention (PCI).

Methods: Patients with AMI who received PCI were consecutively included. The definition of preprocedural hyperuricemia was a SUA level $>7 \mathrm{mg} / \mathrm{dL}(417 \mathrm{mmol} / \mathrm{L})$ in males and $>6 \mathrm{mg} / \mathrm{dL}(357 \mathrm{mmol} / \mathrm{L})$ in females. All-cause mortality was assessed during 2.3-year median follow up period.

Results: One thousand and five patients with AMI undergoing PCI were enrolled in a single center study, 307 (30.5\%) patients had hyperuricemia. After adjusting for potential confounding factors, the multivariable analysis indicated that preprocedural hyperuricemia was related to an increased risk of all-cause mortality during the 2.3-year follow-up (HR: 1.97; 95\% CI: 1.11-3.49; P=0.019).
\end{abstract}

Conclusions: Preprocedural hyperuricemia, independently from chronic kidney disease (CKD), is a significant and independent predictor of long-term mortality for patients with AMI who underwent PCI.

Keywords: Uric acid (UA); acute myocardial infarction (AMI); percutaneous coronary intervention (PCI)

Submitted Oct 08, 2019. Accepted for publication Oct 16, 2019.

doi: $10.21037 /$ atm.2019.10.110

View this article at: http://dx.doi.org/10.21037/atm.2019.10.110

\section{Introduction}

Acute myocardial infarction (AMI) refers to a wide range of clinical manifestations including ST-segment elevation myocardial infarction and non-ST-segment elevation myocardial infarction, which is associated with high morbidity and mortality rates. Thus, identifying poor outcome related to patients with AMI would be of great help as they can be observed with cautions and treated with an optimal preventive schedule.

Uric acid (UA) is the final product of purine metabolism, 
which is metabolized by xanthine oxidase (1). UA have been found to be linked to hypertension (2), diabetes (3), kidney disease (4) and obesity (5). Increased serum UA (SUA) levels may be associated with high level of cardiovascular mortality.

However, it is controversial that whether hyperuricemia is an independent predictor of AMI in patients underwent percutaneous coronary intervention (PCI) (6). A previous study demonstrated that UA may play a role in long-term major adverse outcomes in patients with acute coronary syndrome (ACS) treated with PCI (7). In the context of AMI, data on SUA level and long-term mortality is limited in contemporarily management. The purpose of the present study is to survey the prognostic role of hyperuricemia in patients with AMI underwent PCI during a long-term follow up.

\section{Methods}

\section{Study population}

All patients aged $\geq 18$ with AMI undergoing PCI between April 2009 and December 2013 at Guangdong Cardiovascular Institute, Guangdong Provincial People's Hospital were consecutively enrolled. The exclusion criteria included intravascular administration of a contrast medium within the 7 days before procedure, prescription of metformin in subjects whose estimated glomerular filtration rate (eGFR) was lower than $60 \mathrm{~mL} / \mathrm{min} / 1.73 \mathrm{~m}^{2}$, pregnancy, lactation, usage of $\mathrm{N}$-acetylcysteine, aminoglycosides or any drugs with renal toxicity within a week before procedure (especially nonsteroidal anti-inflammatory drugs during the 48 hours before procedure), allergic to contrast agent, severe complication, kidney transplantation, or end-stage renal failure requiring renal replacement therapy. We also excluded subjects who did not survive the procedure and those who had intra-aortic balloon pump before procedure due to hemodynamic instability. The Ethics Committee of the Guangdong Provincial People's Hospital approved this study, and written informed consent was obtained from each patient. All eligible patients enrolled in this study were followed up through telephone interviews or office visits.

\section{Definitions}

All-cause mortality was defined as any death recorded after the date of enrollment. Chronic kidney disease (CKD) was defined as eGFR $<60 \mathrm{~mL} / \mathrm{min} / 1.73 \mathrm{~m}^{2}$ calculated by the modification of diet in renal disease (MDRD) Formula. What's more, patients with systolic blood pressure $<90 \mathrm{mmHg}$ or diastolic blood pressure $<60 \mathrm{mmHg}$ were considered to suffer hypotension.

\section{Intervention}

All the procedures were conducted by an experienced interventional group and performed according to standard clinical practice guidelines using standard guide catheters, guidewires, balloon catheters, and stents via femoral or radial approach. The contrast dose was at the discretion of the interventional cardiologists.

Eligible patients were divided into 2 groups which is normouricemia or hyperuricemia. Hyperuricemia was defined as a SUA level $>7 \mathrm{mg} / \mathrm{dL}(417 \mathrm{mmol} / \mathrm{L})$ in men and $>6 \mathrm{mg} / \mathrm{dL}(357 \mathrm{mmol} / \mathrm{L})$ in women, as was mentioned in our previous studies $(8,9)$. The use of angiotensinconverting enzyme inhibitors, adrenergic-blocking agents, diuretics, or platelet glycoprotein IIb/IIIa receptor inhibitor (e.g., abciximab) was decided by the care providers and was relied on the routine standards of care recommended by the current guidelines.

\section{Statistical analyses}

Continuous variables with normally distribution were displayed with means \pm standard deviation and compared with one-way ANOVA or $t$-tests. Continuous variables that were nonnormally distributed were presented with median and interquartile ranges and analyzed utilizing Wilcoxon rank-sum tests. Categorical data which were displayed as percentages were analyzed with Fisher exact tests or Pearson Chi-square. All-cause deaths in both groups were assessed with survival analysis. Multivariable cox regression analyses were performed to identify risk factors for longterm mortality. All statistical analyses were performed using SPSS software version 22.0 (SPSS Inc., Chicago, Illinois, USA) and R soft-ware (version 3.1.2; R Core Team, Vienna, Austria).

\section{Results}

\section{Clinical characteristics of patients}

The baseline characteristics of 1,005 AMI patients who underwent PCI are displayed in Table 1. Finally, about 307 (30.5\%) patients were divided into the hyperuricemic 
Table 1 Baseline variables between normouricemic group and hyperuricemic group

\begin{tabular}{|c|c|c|c|}
\hline Variables & Normuricemia $(\mathrm{n}=698)$ & Hyperuricemia $(n=307)$ & $P$ value \\
\hline Age, year & $61.09 \pm 11.89$ & $63.49 \pm 13.18$ & 0.006 \\
\hline Age $\geq 75$ years, $n(\%)$ & $99(14.18)$ & $76(24.76)$ & $<0.0001$ \\
\hline Female, n (\%) & $91(13.04)$ & $65(21.17)$ & 0.001 \\
\hline Hypertension, n (\%) & $322(46.2)$ & $184(59.93)$ & $<0.0001$ \\
\hline Hypotension, n (\%) & $23(4.30)$ & $19(8.88)$ & 0.014 \\
\hline CKD, n (\%) & $202(29.71)$ & $159(53.18)$ & $<0.0001$ \\
\hline Hyperlipidemia, n (\%) & $103(14.76)$ & $50(16.29)$ & 0.5340 \\
\hline \multicolumn{4}{|l|}{ Examination } \\
\hline Uric acid, umol/L & $308.4 \pm 62.72$ & $502.4 \pm 99.83$ & $<0.0001$ \\
\hline Albuminemia, g/L & $33.05 \pm 4.71$ & $33.15 \pm 4.65$ & 0.79 \\
\hline LDL-C, $\mathrm{mmol} / \mathrm{L}$ & $2.99 \pm 1.06$ & $2.92 \pm 1.03$ & 0.42 \\
\hline Total cholesterol, $\mathrm{mmol} / \mathrm{L}$ & $4.62 \pm 1.21$ & $4.62 \pm 1.28$ & 0.9491 \\
\hline $\mathrm{HbA1c}(\%)$ & $6.61 \pm 1.56$ & $6.55 \pm 1.32$ & 0.63 \\
\hline \multicolumn{4}{|l|}{ Medication therapy } \\
\hline Beta-blocker, n (\%) & $580(83.09)$ & $245(79.80)$ & 0.210 \\
\hline
\end{tabular}

CKD, chronic kidney disease; DM, diabetes mellitus; LVEF, left ventricular ejection fraction; LDL-C, low-density lipoprotein cholesterol; ACEI/ARB, angiotensin-converting enzyme inhibitors/angiotensin receptor blocker; CABG, coronary artery bypass grafting.

group and 698 (69.5\%) into the normouricemic group. Higher proportion of women, higher prevalence of hypotension and CKD were also detected in the hyperuricemic group. Patients with higher SUA have lower proportion of left ventricular ejection fraction (LVEF) $\geq 40$ comparing with those with normal SUA.

\section{Hyperuricemia and long-term mortality}

According to the survival analysis (Figure 1), there was significantly different in all-cause mortality between groups.
During the mean follow-up of $2.3 \pm 1.0$ years, we identified a higher incidence of death in the hyperuricemic group. According to the Kaplan-Meier curves, a higher risk of death appeared when hyperuricemia presented.

Results from multivariable cox regression analysis revealed that the risk of death after PCI were significantly $(\mathrm{P}<0.05)$ increased for patients with $\mathrm{CKD}$ and hyperuricemia, while decreased in patients with LVEF $\geq 40$ and prescription of beta-blocker (Table 2). Mortality for patients with hyperuricemia was higher than that of patients with normal SUA (HR: 1.97; 95\% CI: 1.11-3.49; $\mathrm{P}=0.019$ ) in the period 


\section{Page 4 of 6}

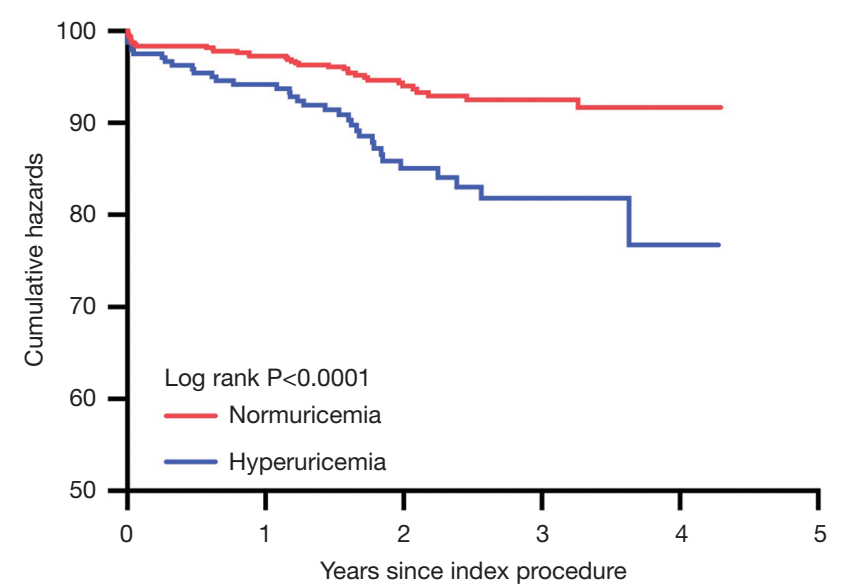

Number of patients at risk

$\begin{array}{lcccc}\text { Normuricemia } & 539 & 268 & 137 & 1 \\ \text { Hyperuricemia } & 220 & 95 & 43 & 1 \\ \text { Year since index procedure } & 1 & 2 & 3 & 4\end{array}$

Figure 1 Kaplan-Meier estimates of mortality according to hyperuricemia. The incidence of mortality was increased in the hyperuricemic group.

Table 2 Predictive factors for mortality according to multivariable Cox regression analysis

\begin{tabular}{lccc}
\hline Variable & HR & $95 \% \mathrm{Cl}$ & P value \\
\hline Hyperuricemia & 1.97 & $1.11-3.49$ & 0.019 \\
Age $\geq 75$ & 1.37 & $0.74-2.54$ & 0.310 \\
Female & 1.05 & $0.52-2.10$ & 0.884 \\
LVEF $\geq 40$ & 0.35 & $0.18-0.68$ & 0.002 \\
CKD & 3.58 & $1.83-7.01$ & 0.001 \\
Hypotension & 1.69 & $0.71-4.01$ & 0.232 \\
Beta-blocker & 0.42 & $0.24-0.77$ & 0.004 \\
ACEl/ARB & 0.52 & $0.25-1.05$ & 0.068 \\
\hline
\end{tabular}

Multivariate Cox regression analysis indicated that the HRs for death in patients with acute myocardial infarction after percutaneous coronary intervention. $\mathrm{Cl}$, confidence interval; $\mathrm{HR}$, hazard ratio; LVEF, left ventricular ejection fraction; CKD, chronic kidney disease; ACEI/ARB, angiotensin-converting enzyme inhibitors/angiotensin receptor blocker.

of follow-up. CKD (HR: 3.58; 95\% CI: 1.83-7.01; P=0.001) was also associated with an increased risk of death, while LVEF $\geq 40$ (HR: 0.35; 95\% CI: 0.18-0.68; $\mathrm{P}=0.002$ ) and Beta-blocker (HR: 0.42; 95\% CI: 0.24-0.77; $\mathrm{P}=0.004$ ) was associated with a decreased risk of death.

\section{Guo et al. Hyperuricemia and long-term mortality in AMI with PCI}

\section{Discussion}

Our present study has shown that preprocedural hyperuricemia was frequently observed in AMI patients underwent PCI and predicted long-term mortality significantly and independently, independently from CKD.

It is believed that UA was linked with cardiovascular disease $(4,10)$. UA is definitely associated with established cardiovascular risk factors such as hypertension, metabolic syndrome, BMI, HDL-c, triglycerides and renal function. However, it was controversial that UA was regarded as an independent cardiovascular risk factor. Even if UA itself may not considered as a direct risk factor for cardiovascular disease, it is also likely to be a predictor for cardiovascular risk and may exacerbate other known risk factors. In fact, there are studies had shown that UA takes effect in hypertension (2), diabetes (3) and obesity (5). A meta-analysis of 14 prospective cohort studies (341,389 patients) showed hyperuricemia might be correlated with an increased risk of coronary heart disease death (RR: 1.14, 95\% CI: 1.06 to $1.23, \mathrm{P}=0.342)$ and an increased risk of allcause mortality (RR: $1.20,95 \% \mathrm{CI}: 1.13$ to $1.28, \mathrm{P}<0.001$ ). Moreover, for every $1 \mathrm{mg} / \mathrm{dL}$ increase in SUA levels, the overall risk of coronary heart disease and all-cause mortality increased by $20 \%$ and $9 \%$, respectively (11).

An independent association of UA in patients presenting with ACS including myocardial infarction was concerned about short-term outcome. Magnoni and his colleagues has reported that in patients with ACS, uricemia levels above $6.0 \mathrm{mg} / \mathrm{dL}$ were associated with in-hospital mortality, independently from ethnicity and renal function (12). The previous study has shown that UA $>6.5 \mathrm{mg} / \mathrm{dL}$ were observed in the $21.5 \%$ of the patients and were independently linked with in-hospital mortality in STEMI patients undergoing PCI (13).

The results of above studies suggest that UA has prognostic value for mortality in patients with AMI. However, it is only confined to short-term prognosis. Concerning the relation of UA with mid-term and longterm outcome, data is scarce. One study enrolled a total of 27,959 patients with ACS and the mean follow-up of 1 year (data from PLATO and TRACER studies) showed a risk of cardiovascular death in patients with hyperuricemia (HR: 1.09, 95\% CI: $1.05-1.14, \mathrm{P}<0.0001)$ and all-cause mortality risk (HR: $1.10,95 \%$ CI: $1.06-1.14, \mathrm{P}<0.0001$ ) were higher, and hyperuricemia was an independent risk factor (14). Another multicenter study has mean follow-up for 2 years of 2,249 patients with STMEI showed that hyperuricemia 
was independently associated with cardiovascular death after primary PCI (15). In a longer follow-up (mean 5.5 years) study, Tscharre was enrolled in 1,215 patients with ACS who underwent PCI. The results also showed a high risk of 1.6-fold increased risk of cardiovascular death (HR: 1.606, 95\% CI: 1.157-2.228, P=0.005) (7). A Japanese study included 1,124 AMI patients has reported that similar levels of UA (more than $7.5 \mathrm{mg} / \mathrm{dL}$ ) led to the optimal cut-off to forecast long-term mortality (16).

In our study, we found hyperuricemia was associated with long-term mortality during 2.3-year median follow up period, which effectively support hyperuricemia is a risk factor for patients with AMI. Renal insufficiency which is one of cardiovascular risk factors is closely bound up with coronary heart disease and cardiovascular mortality. Kowalczyk et al. have shown that UA concentrations predict short- and long-term mortality in patients with renal insufficiency who have myocardial infarction undergoing PCI (17). After adjusting CKD, the results from our data was also lined with long-term mortality.

Basic research has found that UA is associated with the development of arteriosclerosis and cardiovascular disease. Intracellular and mitochondrial UA aggravates arteriosclerosis by releasing free radicals and inducing oxidative stress. UA may also stimulate the expression of inflammatory factors to induce vascular inflammation, such as vascular smooth muscle cell mononuclear chemoattractant protein-1 (MCP-1) $(18,19)$. In addition, UA was found to be able to reduce NO synthesis and release, which may cause endothelial dysfunction and promote lipid plate formation; damage cardiac microvessels, reduce coronary blood flow reserve, etc. $(20,21)$, and contribute to the formation of atherosclerosis.

Our current study is subject to the following limitations. First, the study was an observational study and included a small population in a single center. It needs a large, multicenter trial to confirm our results. Second, long-term mortality was predicted by the use of a single preprocedural measurement of UA in our study. However, several studies have used a baseline measurement of UA in the past.

\section{Conclusions}

Our study found hyperuricemia were associated with longterm mortality in patients with AMI undergoing PCI after adjusting renal function.

\section{Acknowledgments}

Funding: This study was supported by the Medical Research Fund Project of Guangdong Province (grant number C2017054); the Science and Technology Planning Project of Guangdong Province (grant No. 2014B070706010); the National Science Foundation for Young Scientist of China (grant No. 81500520).

\section{Footnote}

Conflicts of Interest: The authors have no conflicts of interest to declare.

Ethical Statement: The authors are accountable for all aspects of the work in ensuring that questions related to the accuracy or integrity of any part of the work are appropriately investigated and resolved. All data in the study was collected following approval by the ethics committees (approval number: GDREC2010112H) and was registered on the ClinicalTrials.gov with the registration ID NCT01400295. And written informed consent was obtained from each patient.

\section{References}

1. Kanbay M, Segal M, Afsar B, et al. The role of uric acid in the pathogenesis of human cardiovascular disease. Heart 2013;99:759-66.

2. Shankar A, Klein R, Klein BE, et al. The association between serum uric acid level and long-term incidence of hypertension: Population-based cohort study. J Hum Hypertens 2006;20:937-45.

3. Dehghan A, van Hoek M, Sijbrands EJ, et al. High serum uric acid as a novel risk factor for type 2 diabetes. Diabetes Care 2008;31:361-2.

4. Borghi C, Rosei EA, Bardin T, et al. Serum uric acid and the risk of cardiovascular and renal disease. J Hypertens 2015;33:1729-41; discussion 1741 .

5. Masuo K, Kawaguchi H, Mikami H, et al. Serum uric acid and plasma norepinephrine concentrations predict subsequent weight gain and blood pressure elevation. Hypertension 2003;42:474-80.

6. Culleton BF, Larson MG, Kannel WB, et al. Serum uric acid and risk for cardiovascular disease and death: the Framingham Heart Study. Ann Intern Med 1999;131:7-13.

7. Tscharre M, Herman R, Rohla M, et al. Uric acid is 
associated with long-term adverse cardiovascular outcomes in patients with acute coronary syndrome undergoing percutaneous coronary intervention. Atherosclerosis 2018;270:173-9.

8. Guo W, Liu Y, Chen JY, et al. Hyperuricemia Is an Independent Predictor of Contrast-Induced Acute Kidney Injury and Mortality in Patients Undergoing Percutaneous Coronary Intervention. Angiology 2015;66:721-6.

9. Liu Y, Tan N, Chen J, et al. The relationship between hyperuricemia and the risk of contrast-induced acute kidney injury after percutaneous coronary intervention in patients with relatively normal serum creatinine. Clinics (Sao Paulo) 2013;68:19-25.

10. Savarese G, Ferri C, Trimarco B, et al. Changes in serum uric acid levels and cardiovascular events: a meta-analysis. Nutr Metab Cardiovasc Dis 2013;23:707-14.

11. Zuo T, Liu X, Jiang L, et al. Hyperuricemia and coronary heart disease mortality: a meta-analysis of prospective cohort studies. BMC Cardiovasc Disord 2016;16:207.

12. Magnoni M, Berteotti M, Ceriotti F, et al. Serum uric acid on admission predicts in-hospital mortality in patients with acute coronary syndrome. Int J Cardiol 2017;240:25-9.

13. Lazzeri C, Valente S, Chiostri M, et al. Uric acid in the acute phase of ST elevation myocardial infarction submitted to primary PCI: its prognostic role and relation with inflammatory markers: a single center experience. Int J Cardiol 2010;138:206-9.

14. Pagidipati NJ, Hess CN, Clare RM, et al. An examination of the relationship between serum uric acid level, a clinical history of gout, and cardiovascular outcomes among patients with acute coronary syndrome. Am Heart J

Cite this article as: Guo W, Yang D, Wu D, Liu H, Chen S, Liu J, Lei L, Liu Y, Rao L, Zhang L; The RESCIND Group. Hyperuricemia and long-term mortality in patients with acute myocardial infarction undergoing percutaneous coronary intervention. Ann Transl Med 2019;7(22):636. doi: 10.21037/ atm.2019.10.110
2017;187:53-61.

15. Kaya MG, Uyarel H, Akpek M, et al. Prognostic value of uric acid in patients with ST-elevated myocardial infarction undergoing primary coronary intervention. Am J Cardiol 2012;109:486-91.

16. Kojima S, Sakamoto T, Ishihara M, et al. Prognostic usefulness of serum uric acid after acute myocardial infarction (the Japanese Acute Coronary Syndrome Study). Am J Cardiol 2005;96:489-95.

17. Kowalczyk J, Francuz P, Swoboda R, et al. Prognostic significance of hyperuricemia in patients with different types of renal dysfunction and acute myocardial infarction treated with percutaneous coronary intervention. Nephron Clin Pract 2010;116:c114-22.

18. Cheng TH, Lin JW, Chao HH, et al. Uric acid activates extracellular signal-regulated kinases and thereafter endothelin-1 expression in rat cardiac fibroblasts. Int J Cardiol 2010;139:42-9.

19. Spiga R, Marini MA, Mancuso E, et al. Uric Acid Is Associated With Inflammatory Biomarkers and Induces Inflammation Via Activating the NF-kappaB Signaling Pathway in HepG2 Cells. Arterioscler Thromb Vasc Biol 2017;37:1241-9.

20. Kanellis J, Watanabe S, Li JH, et al. Uric acid stimulates monocyte chemoattractant protein-1 production in vascular smooth muscle cells via mitogen-activated protein kinase and cyclooxygenase-2. Hypertension 2003;41:1287-93.

21. Akpek M, Kaya MG, Uyarel H, et al. The association of serum uric acid levels on coronary flow in patients with STEMI undergoing primary PCI. Atherosclerosis 2011;219:334-41. 